\title{
Investigating the short-term impact of cognitive-behavioral therapy (CBT) on quality of life in Persian patients with rheumatoid arthritis: the heterogeneous impact on Arthritis Impact Measurement Scales (AIMS-2)
}

\author{
Maassoumeh Akhlaghi, Seyedeh Tahereh Faezi, Pedram Paragomi", Farimah Ashofteh, Pari Alinejad, Neda \\ Hatami, Laleh Ghadirian \\ Rheumatology Research Center, Shariati Hospital, Tehran University of Medical Sciences, Tehran, Iran
}

\begin{abstract}
This study evaluated the effect of cognitive-behavioral therapy (CBT) compared with other treatments on improving the quality of life in rheumatoid arthritis (RA) patients. This study was carried out in a rheumatologic referral clinic at an academic hospital. RA patients were categorized in three subgroups: cognitive behavioral therapy group, educational therapy group, and conventional treatment as the control group. Quality of life was assessed with the Arthritis Impact Measurement Scales (AIMS-2) questionnaire. The CBT subgroup $(n=30)$ received cognitive-behavioral treatment with 7 two-hour sessions twice a week; the educational therapy group $(n=30)$ received education about nutrition and osteoporosis, while controls $(n=30)$ received conventional RA medical treatment. Outcomes were gathered in 14 domains of AIMS-2 including activity, walking, pain, self-care, social activity, depression, and anxiety. The 90 studied patients comprised 72 female patients $(80.0 \%)$ with a mean age of 41.7 years. The degree of improvement in physical activity $(p=0.2)$, hand/finger function $(p=0.18)$, arm function $(p=0.28)$, social activity $(p=0.6)$, satisfaction $(p=0.05)$, household tasks $(p=0.9)$, health perception $(p=0.3)$, self-care ability $(p=0.59)$ showed no significant difference between the three subgroups. Moreover, CBT was effective in improving mood, ability to walking and bend, working, reducing pain and tension, and these effects were independent of age, gender, or education. Additionally, education about nutrition in RA patients improved their ability to work and their mood, and it effectively reduced tension. Based on the findings, CBT is a recommended modality adjunct to RA medical treatment. CBT is specifically beneficial for patients with depressed mood, problems in walking, bending or working, and in subjects who are suffering psychosocial tension.
\end{abstract}

Keywords: AIMS-2, cognitive-behavioral therapy, psychotherapy, quality of life, rheumatoid arthritis.

\section{Introduction}

Rheumatoid arthritis (RA) is the most common chronic inflammatory arthritis with no known cause that leads to joint destruction and disability [1, 2]. RA is distributed in all ethnic groups all over the world [3].

The total prevalence of disease has been estimated to be $0.57 \%$ for females and $0.14 \%$ for males [4]. RA imposes considerable social and economic burdens because of its chronic nature, subsequent handicap, and long-term disability [5]. In the USA, the average capitation of medical costs for bone and joint patients in 1996 was \$3578; the total spent was \$193 billion, which was equivalent to $2.5 \%$ of the gross domestic product.

Treatments for RA include medications, biological treatments, and surgery [6]. In spite of a good understanding of the pathogenesis of this disease, the availability of criteria and newer diagnostic tests, earlier and more accurate diagnosis, and the considerable number of novel treatments, RA still causes many disabilities; some patients are even in danger of an early death [7-9].

Cognitive Behavioral Therapy (CBT) is known as an efficient short-term goal-oriented modality of psychotherapy [10]. Albeit primarily applied for mood disorders, the role of CBT has expanded to include states of chronic pain [10].

In combination with medical treatments, CBT has been proposed as an integral therapeutic modality in RA [11]. CBT has led to positive outcomes by enhancing coping capacities and reducing catastrophic thinking about pain $[12,13]$. Many studies have been done on RA and other rheumatologic diseases which have investigated the effects of teaching patients about treatment processes and side effects of diseases $[14,15]$.

\footnotetext{
* Corresponding Authors: Pedram Paragomi, Emails: pedram.paragomi@gmail.com, Tel: +98 21 84902405, Fax: +98 2184902405 Received: 07 December 2016; Accepted: 04 July 2017
} 
However, there are some questions about the effectiveness of CBT on significant results and the underlying mechanism involved in the ameliorative function of CBT. A pilot study by Ferguson et al. proposed a positive impact of CBT on RA patients' adherence to treatment [16]. Considering the remaining ambiguities in past studies, the heavy burden of RA in Iran and the cost and side effects of treatment, the current study was designed to more precisely explore the effect of this type of psychotherapy on the lifestyle of Iranian rheumatoid arthritis patients. Researchers painstakingly tried to find the alteration pattern of various domains of AIMS-2 after CBT training.

\section{Patients and Methods}

This case control study was conducted at the rheumatology clinic of Shariati Hospital, Tehran, Iran. Diagnoses of RA were confirmed by a rheumatologist based on American College of Rheumatology (ACR) criteria. Enrolled patients were residents of Tehran and were in the age range of 16-65. The patients were instructed to contact the research team by phone on a regular basis. Exclusion criteria were the presence of comorbidities which could potentially affect the patient's quality of life, residence in homes for the elderly or any other nursing centers, and having a cognitive behavioral disorder. Moreover, patients with a disability that prevented the rheumatologist from educating them or patients with American Rheumatism Association criteria for functional class (ARA) $>2$ were not eligible for this study.

\section{Data collection instrument}

The Arthritis Impact Measure Scale (AIMS-2) questionnaire was applied to assess the patients' quality of life. AIMS-2 evaluates the twelve factors of mobility, hand and finger function, walking, arm function, pain, self-care, daily household tasks, social activities, social support, work, depression, and stress. It also collects personal information, the address and telephone number of the patient or his/her companions who give the information. After questions asking for demographic and contact information, questions assessing life quality in RA patients ensue.

The questionnaire consisted of 78 sections. The first 57 questions covered the 12 above-mentioned qualityof-life factors. The $58^{\text {th }}$ item that was applied specifically for this study evaluated the satisfaction scale of each of these twelve cases. The score of each case was registered between 0-10 (0 indicating a good situation of health and 10 indicating a weak situation). Hence, lower scores on the AIMS-2 indicate a higher quality level

\section{Validation of Persian version of AIMS-2}

This questionnaire was translated once into Persian, and then it was back-translated to English by a translator with no knowledge of this research project. The backtranslated version was compared with the original questionnaire. The test and re-test were compared by sending the questionnaires after 4 weeks to be completed again by the patients. No data was missing. Meanwhile, three rheumatologists evaluated the questionnaire for content validity.

To examine the face validity prior to launching the study, the questionnaire was completed by 20 patients. A Cronbach's $\alpha$ coefficient of 0.84 revealed acceptable internal consistency of the adapted AIMS-2.

\section{Patient enrollment and data collection}

The enrolled cases were entered into the study through convenience sampling by blocked random allocation. Ninety participants were divided into three subgroups: CBT (CBT+), non-CBT (CBT-) and Control. Double blinding was also considered in the management of patients and allocator.

After signing an informed consent form, eligible patients completed the questionnaire in the presence of the main researcher who could answer the presumable problems.

The Control group received conventional RA treatment, while the CBT intervention group (CBT+ subgroup) attended training (7 two-hour sessions, twice a week). The non-CBT group (CBT- subgroup) got a training plan about diet and instructions for avoiding drug-induced osteoporosis. On the first day of the study and in the last session of training, all participants completed the AIMS-2 forms.

\section{Calculation of sample size}

The sample size was calculated based on a reference randomized clinical trial by Sharpe et al. (Power $=80 \%$ and $a=0.005)$ [17]. The calculated sample size was 30 patients for each subgroup (CBT+, CBT-, and Control). Overall, 90 RA cases were enrolled in the current study.

\section{Data analysis}

Mean and standard deviation were used to indicate descriptive statistical variables, and absolute frequency and prevalence were used to indicate qualitative statistics. For descriptive analysis based on objectives, one-way ANOVA, Mann-Whitney U, independentsamples $\mathrm{T}$ test, Chi square, and Kruskal-Wallis tests were used. Collected data was analyzed using SPSS 
22.0 (Chicago, IL). A p-value $=0.05$ was considered significant.

\section{Ethical issues}

The study proposal was approved by the Ethics Committee of Tehran University of Medical Sciences. All participants were required to sign an informed consent form. The research team was committed to absolute confidentiality of patient information. After the completion of data gathering, codes were used instead of assigned names.

\section{Results}

The studied population comprised $72(80 \%)$ female and
18 (20\%) male cases. There was no significant difference in age, gender, or education level among the three groups. The demographic items of the studied population are summarized in Table 1.

\section{Comparing pre-CBT AIMS-2 scores}

All parameters were compared between the three subgroups at the beginning of the study and prior to undertaking interventions. No significant difference was noted in the various AIMS-2 features, except in selfcare. CBT- patients had the highest self-care score, while CBT+ patients had the lowest self-care score $(p=0.001)$. The pre-CBT AIMS-2 scores are tabulated in Table 2 .

Table 1. The demographic characteristic of the RA patients in three intervention subgroups

\begin{tabular}{|c|c|c|c|c|}
\hline \multirow{2}{*}{ Demographic variable } & \multicolumn{3}{|c|}{ Subgroups } & \multirow[b]{2}{*}{ p-value } \\
\hline & CBT+ & CBT- & Control & \\
\hline Mean Age & $\begin{array}{c}44.0 \\
\text { (95\% CI: 40-47) }\end{array}$ & $\begin{array}{c}45.7 \\
(95 \% \text { CI: 41-49) }\end{array}$ & $\begin{array}{c}45.4 \\
\text { (95\% CI: 43-47) }\end{array}$ & 0.78 \\
\hline \multicolumn{5}{|c|}{ Sex } \\
\hline Male & $4(13.4 \%)$ & $6(20.0 \%)$ & $8(26.8 \%)$ & \multirow{2}{*}{0.44} \\
\hline Female & $26(86.6 \%)$ & $24(80.0 \%)$ & $22(73.2 \%)$ & \\
\hline \multicolumn{5}{|c|}{ Years of education } \\
\hline 10> & $12(40.0 \%)$ & $10(33.4 \%)$ & $13(43.3 \%)$ & \multirow{3}{*}{0.66} \\
\hline $10-12$ & $16(53.4 \%)$ & $16(53.4 \%)$ & $16(53.4 \%)$ & \\
\hline$>12$ & $2(6.6 \%)$ & $4(13.2 \%)$ & $1(3.3 \%)$ & \\
\hline
\end{tabular}

CI: Confidence Interval

Table 2. Comparing mean scores of variables in three intervention subgroups of RA patients before the interventions

\begin{tabular}{|c|c|c|c|c|}
\hline \multirow{2}{*}{ AIMS-2 Variables } & \multicolumn{3}{|c|}{ Intervention Group } & \multirow{2}{*}{ P-value } \\
\hline & CBT+ & CBT- & Control & \\
\hline Physical ability & $3.08 \pm 2.04^{*}$ & $2.93 \pm 1.58^{*}$ & $3.33 \pm 1.23^{*}$ & 0.64 \\
\hline Walking and Bending & $5.0 \pm 1.98^{\pi}$ & $4.3 \pm 1.55^{\pi}$ & $4.5 \pm 1.30^{\pi}$ & 0.38 \\
\hline Upper extremities' function & $1.80 \pm 1.87^{*}$ & $2.03 \pm 2.49^{*}$ & $2.73 \pm 1.54^{*}$ & 0.18 \\
\hline Social activity & $4.73 \pm 1.11^{*}$ & $4.47 \pm 1.03^{*}$ & $4.95 \pm 1.07^{*}$ & 0.22 \\
\hline Work & $4.2 \pm 2.32^{\pi}$ & $3.9 \pm 1.23^{\pi}$ & $3.7 .1 .07^{\pi}$ & 0.69 \\
\hline Pain & $5.28 \pm 2.11^{*}$ & $4.27 \pm 2.22^{*}$ & $4.15 \pm 1.35^{*}$ & 0.04 \\
\hline Stress & $5.43 \pm 1.79^{*}$ & $4.70 \pm 1.25^{*}$ & $4.52 \pm 1.76^{*}$ & 0.07 \\
\hline Mood & $4.50 \pm 1.49^{*}$ & $4.82 \pm 1.58^{*}$ & $4.03 \pm 1.22^{*}$ & 0.07 \\
\hline Satisfaction & $3.81 \pm 1.78^{*}$ & $3.58 \pm 1.59^{*}$ & $4.63 \pm 1.75^{*}$ & 0.05 \\
\hline Family protection & $3.77 \pm 2.51^{*}$ & $3.50 \pm 2.59^{*}$ & $2.96 \pm 2.27^{*}$ & 0.43 \\
\hline Household task & $2.3 \pm 1.05^{\pi}$ & $2.1 \pm 1.43^{\pi}$ & $2.8 \pm 1.02^{\pi}$ & 0.42 \\
\hline Self-Care & $2.31 \pm 1.20^{*}$ & $4.12 \pm 2.66^{*}$ & $2.77 \pm 1.32^{*}$ & 0.001 \\
\hline
\end{tabular}

"Scores presented as Mean \pm Standard Deviation

${ }^{\pi}$ Scores presented as Median \pm interquartile range

AIMS-2: Arthritis Impact Measurement Scales 


\section{Comparing post-CBT AIMS-2 scores}

The degrees of improvement in physical activity $(p=0.2)$, hand and finger function $(p=0.18)$, arm function $(p=0.28)$, social activity $(p=0.6)$, satisfaction $(p=0.05)$, household tasks $(p=0.9)$, health perception $(p=0.3)$, and self-care ability $(p=0.59)$, showed no significant differences among the three subgroups. Meanwhile, a significant improvement in walking and bending ability $(p=0.04)$ was detected where the CBT+ subgroup had an improved score in comparison with the controls $(p=0.02)$. The improvement-in-work item showed a significant difference among the three groups $(p=0.004)$. Post-hoc analysis revealed that both CBT+ $(p=0.001)$ and CBT- groups $(p=0.01)$ had significantly improved scores in comparison with the Control group.

A significant difference in the pain item was detected among the three subgroups $(p=0.04)$. A twoby-two comparison revealed significantly lower scores in CBT+ patients than in the Controls $(p=0.008)$. Stress reduction showed a significant difference in the three groups $(p=0.001)$. A significant improvement was noted in the CBT+ $(p=0.004)$ and CBT- subgroups $(p=0.001)$ compared with the Controls.

Moreover, mood improvement showed a significant difference among the three subgroups $(p=0.02)$. Posthoc analysis unraveled significant improvement in both the CBT+ $(p=0.024)$ and the CBT- $(p=0.005)$ subgroup in comparison with the Controls.

\section{The influence of age, gender, and education on the AIMS-2 scores of the CBT+ subgroup}

There was no significant difference in the effect of CBT on mobility $(p=0.5)$, walking and bending $(p=0.8)$, hand and finger function $(p=0.9)$, arm function $(p=0.6)$, pain $(p=0.7)$, stress reduction $(p=0.9)$, mood improvement $(p=0.9)$, satisfaction increase $(p=0.3)$, the perception of health $(p=0.3)$, or the need for family support $(p=0.2)$ among different age groups.

Gender had no influence on the effect of CBT on mobility improvement $(p=0.5)$, walking and bending $(p=0.5)$, hand and finger function $(p=0.74)$, arm function $(p=0.65)$, self care $(p=0.09)$, household tasks improvement $(\mathrm{p}=0.13)$, social activity improvement $(p=0.71)$, arthritis pain reduction $(p=0.13)$, working ability increase $(p=0.57)$, stress reduction $(p=0.95)$, mood improvement $(p=0.91)$, satisfaction increase $(p=0.74)$, perception of health $(p=0.46)$, or decreasing the need for family support $(p=0.72)$.

Education level had no influence on the effect of CBT on mobility improvement $(p=0.61)$, walking and bending $(p=0.1)$, hand and finger function $(p=0.22)$, arm function $(p=0.66)$, self care $(p=0.74)$, stress reduction $(p=0.44)$, mood improvement $(p=0.27)$, satisfaction increase $(p=0.22)$, perception of health $(p=0.98)$, or the need for family support $(p=0.69)$.

\section{Discussion}

CBT is known as an efficient short-term goal-oriented modality of psychotherapy [10]. Previous studies have suggested the sustained influence of CBT on patients in states of chronic pain [15]. Results can be achieved through a 10 - to 20 -session schedule by targeting subjects' thought patterns and the link between thoughts and feelings [10]. CBT induces a robust impact on selfefficacy, adaptive coping, pain-related behaviors, and catastrophic thinking $[10,18,19]$. However, there are numerous domains of quality of life which need to be exclusively analyzed in RA patients.

In the current study, the demographic characteristics of patients including age, gender prevalence, and education level that could influence the results were similar in the three subgroups. There was no significant difference among the three subgroups in fourteen preCBT AIMS-2 items. These studied axes included mobility, walking and bending ability, hand and finger function, arm function, social activity, work, pain, tension level, mood, satisfaction, perception of health, need for family support, and household tasks improvement. However, self-care ability at the pre-CBT stage demonstrated a significant variance. This significant difference stemmed from the comparison between controls and both CBT+ and CBT-groups.

No mobility improvement was seen in CBT+ patients. This result opposes those of a number of previous studies $[14,17,20,21]$. Moreover, no improvement in social activity was observed after CBT, which was in contrast to past studies $[17,22,23]$.

There was no significant difference between patient self-care and their perception of health; this was in contrast to a study by Sharp [24]. It is noteworthy that the CBT+ cases had significantly better baseline selfcare scores. This intriguing result reveals overall improvement in all three subgroups; however, the changes were more pronounced in the CBT- and Control patients.

There was a significant difference in walking and bending improvement, work, pain, and tension level among the three subgroups, and this result was similar to previous studies $[11,14,16,20,22,25]$. Conversely, a number of studies have indicated better function, depression, and anxiety in controls in comparison with the intervention group $[17,24]$. 
In the study, by Freeman et al. [26], CBT was compared to control group. Interestingly, their results were better in controls in the short-term, while in longterm, there was no significant difference between the two subgroups [26]. The difference in this study can be attributed to the difference in enrollment of newlydiagnosed patients. Interestingly, patients who were randomized to receive CBT- trainings improved in work ability, stress reduction, and mood improvement. This positive outcome may have resulted from the nonspecific impact of increased therapist care, as a wellorganized therapist-patient relationship was established in CBT- patients.

Based on the results, CBT training induced a heterogeneous impact on various aspects of AIMS-2 in RA patients. CBT improved walking and bending activity, work ability, pain, stress, and mood. Thus, CBT is recommended to RA patients as an adjunct to standard clinical management of the disease. Of note, the premise of CBT implementation is extended to RA patients regardless of their age, gender, or education level.

Future longitudinal studies are warranted to examine the long-term impact of CBT intervention in RA cohort as well as the cost-effectiveness of this modality.

\section{Conflicts of interest}

The authors declare no conflicts of interest.

\section{References}

1. Luqmani R, et al., British society for rheumatology and British health professionals in rheumatology guideline for the management of rheumatoid arthritis (the first two years). Rheumatology 2006; 45(9): 1167-9. doi: 10.1093/rheumatology/kel215b.

2. Felson DT, Anderson JJ, Boers $\mathrm{M}$, Bombardier C, Chernoff $\mathrm{M}$ Fried B, et al., The American College of Rheumatology preliminary core set of disease activity measures for rheumatoid arthritis clinical trials. The Committee on Outcome Measures in Rheumatoid Arthritis Clinical Trials. Arthritis Rheum 1993; 36(6): 729-40. doi: 10.1002/art. 1780360601.

3. Alamanos Y, Drosos A.A Epidemiology of adult rheumatoid arthritis. Autoimmun Rev 2005; 4(3): 130-6. doi: 10.1016/j.autrev.2004.09.002.

4. Lawrence RC, Helmick CG, Arnett FC, Deyo RA, Felson DT, Giannini EH, et al. Estimates of the prevalence of arthritis and selected musculoskeletal disorders in the United States. Arthritis Rheum 1998; 41(5): 778-99. doi: 10.1002/1529-0131 (199805)41:5<778::aid-art4>3.0. co;2-v.

5. Creed F., Psychological disorders in rheumatoid arthritis: a growing consensus? Ann Rheum Dis 1990; 49(10): 808-12. doi: 10.1136/ard.49.10.808.

6. Strand V, Singh JA. Improved health-related quality of life with effective disease-modifying antirheumatic drugs: evidence from randomized controlled trials. Am J Manag Care 2007; 13(9): S237-51.

7. Maradit-Kremers H, Crowson CS, Nicola PJ, Ballman KV, Roger VL, Jacobsen SJ, et al. Increased unrecognized coronary heart disease and sudden deaths in rheumatoid arthritis: a populationbased cohort study. Arthritis Rheum 2005; 52(2): 402-11. doi: 10.1002/art.20853.

8. Solomon DH, Kremer J, Curtis JR, Hochberg MC, Reed G, Tsao $\mathrm{P}$, et al. Explaining the cardiovascular risk associated with rheumatoid arthritis: traditional risk factors versus markers of rheumatoid arthritis severity. Ann Rheum Dis 2010: 69(11): 1920-5. doi: 10.1136/ard. 2009.122226.

9. Meek IL, Vonkeman HE, van de Laar M.A. Cardiovascular case fatality in rheumatoid arthritis is decreasing; first prospective analysis of a current low disease activity rheumatoid arthritis cohort and review of the literature. BMC Musculoskelet Disord 2014; 15(1): 142. doi: 10.1186/1471-2474-15-142.

10. Bennett R, Nelson D. Cognitive behavioral therapy for fibromyalgia. Nat Clin Pract Rheumatol 2006; 2(8): 416-24. doi: 10.1038/ncprheum0245.

11. Sharpe L, Sensky T, Timberlake $\mathrm{N}$, Ryan B, Brewin CR, Allard S, et al. A blind, randomized, controlled trial of cognitive- behavioural intervention for patients with recent onset rheumatoid arthritis: preventing psychological and physical morbidity. Pain 2001; 89(2-3): 275-83. doi: 10.1016/s0304-3959 (00)00379-1

12. Kashikar-Zuck S, Sil S, LynchJordan AM, Ting TV, Peugh J, Schikler KN, et al. Changes in pain coping, catastrophizing, and coping efficacy after cognitivebehavioral therapy in children and adolescents with juvenile fibromyalgia. J Pain 2013; 14(5): 492-501. doi: 10.1016/j.jpain. 2012.12.019.

13. Libby CJ, Glenwick, D.S. Protective and exacerbating factors in children and adolescents with fibromyalgia. Rehabil Psychol 2010; 55(2): 151-8.

14. Hewlett S, Ambler N, Almeida C, Cliss A, Hammond A, Kitchen K, et al. Self-management of fatigue in rheumatoid arthritis: a randomised controlled trial of group cognitive-behavioural therapy. Ann Rheum Dis 2011; 70(6): 1060-7. doi: 10.1136/ard 2010.144691.

15. Kashikar-Zuck S, Ting TV, Arnold LM, Bean J, Powers SW, Graham TB, et al. Cognitive behavioral therapy for the treatment of juvenile fibromyalgia: a multisite, singleblind, randomized, controlled clinical trial. Arthritis Rheum 2012; 64(1): 297-305. doi: 10.1002/art.30644.

16. Ferguson A, Ibrahim FA, Thomas 
V, Weinman J, Simpson C, Cope $\mathrm{AP}$, et al. Improving medication adherence in rheumatoid arthritis (RA): a pilot study. Psychol Health Med 2015; 20(7): 781-89. doi: $\quad 10.1080 / 13548506.2015$. 1009917.

17. Sharpe L, Sensky T, Timberlake N, Ryan B, Allard S. et al. Longterm efficacy of a cognitive behavioural treatment from a randomized controlled trial for patients recently diagnosed with rheumatoid arthritis. Rheumatology 2003; 42(3): 43541. doi: 10.1093/rheumatology/ keg144.

18. Lynch AM, Kashikar-Zuck S Goldschneider KR, Jones BA, et al., Psychosocial risks for disability in children with chronic back pain. J Pain 2006; 7(4): 244-51. doi: 10.1016/j.jpain.2005. 11.001.

19. Nielson WR, Jensen MP. Relationship between changes in coping and treatment outcome in patients with Fibromyalgia
Syndrome. Pain 2004; 109(3): 233-41. doi: 10.1016/j.pain.2004. 01.002 .

20. Basler HD. Group treatment for pain and discomfort. Patient Educ Couns 1993; 20(2-3): 167 75. doi: 10.1016/0738-3991(93) 90130-o.

21. Astin JA, Beckner W, Soeken K, Hochberg MC, Berman B, et al. Psychological interventions for rheumatoid arthritis: a metaanalysis of randomized controlled trials. Arthritis Rheum 2002; 47(3): 291-302. doi: 10.1002/art. 10416.

22. Sharpe L, Sensky T, Allard S. The course of depression in recent onset rheumatoid arthritis: the predictive role of disability, illness perceptions, pain and coping. J Psychosom Res 2001; 51(6): 713-9. doi: 10.1016/s00223999(01)00266-5.

23. Barsky AJ, Ahern DK, Orav EJ, Nestoriuc Y, Liang MH, Berman IT, et al. A randomized trial of three psychosocial treatments for the symptoms of rheumatoid arthritis. Semin Arthritis Rheum 2010; 40(3): 222-32. doi: $10.1016 /$ j.semarthrit. 2010.04.001.

24. Sharpe L, Sensky T, Timberlake $\mathrm{N}$, Allard S, Brewin CR, et al. The role of cognitive behavioural therapy in facilitating adaptation to illness in rheumatoid arthritis: A case series. Behavioural and Cognitive Psychotherapy 2001; 29(03): 303-309. doi: 10.1017/ s1352465801003046.

25. Dissanayake RK, Bertouch JV. Psychosocial interventions as adjunct therapy for patients with rheumatoid arthritis: a systematic review. Int J Rheum Dis 2010; 13(4): 324-34. doi: 10.1111/j. 1756-185x.2010.01563.x.

26. Freeman $\mathrm{K}$, Hammond $\mathrm{A}$, Lincoln N.B. Use of cognitivebehavioural arthritis education programmes in newly diagnosed rheumatoid arthritis. Clin Rehabil 2002; 16(8): 828-36. doi: $10.1191 / 0269215502 \mathrm{cr} 565$ oa. 